\title{
EQUILATERAL QUANTUM GRAPHS AND BOUNDARY TRIPLES
}

\author{
OLAF POST
}

\begin{abstract}
The aim of the present paper is to analyse the spectrum of Laplace and Dirac type operators on metric graphs. In particular, we show for equilateral graphs how the spectrum (up to exceptional eigenvalues) can be described by a natural generalisation of the discrete Laplace operator on the underlying graph. These generalised Laplacians are necessary in order to cover general vertex conditions on the metric graph. In case of the standard (also named "Kirchhoff") conditions, the discrete operator is the usual combinatorial Laplacian.
\end{abstract}

\section{INTRODUCTION}

A quantum graph is by definition a family of ordinary differential operators acting on each edge $e$ considered as interval $\left(0, \ell_{e}\right)$ of length $\ell_{e}>0$ with boundary conditions at the vertices making the global operator self-adjoint. An equilateral graph is a metric graph where all lengths (inverse edge weights) are the same.

Quantum graphs are believed to play an intermediate role between difference operators on discrete graphs and partial differential operators on manifolds. On the one hand, they are a good approximation of partial differential operators on manifolds or open sets close to the graph (see e.g. [P06, EP07] and the references therein). On the other hand, solving a system of ODEs reduces in many cases to a discrete problem on the combinatorial graph. For more details on quantum graphs we refer to [BCFK06, KoS06, K04, K05] and the references therein.

Boundary triples were originally introduced in order to treat boundary conditions for partial differential operators (see [V63]) and to generalise Green's formula. Nowadays, they became a convenient tool to deal with self-adjoint extensions of closed operators. In particular, boundary triples have been used for quantum graphs in [Pa06, BGP06, BGP07], especially to establish a relation between the quantum and combinatorial graph spectrum. Most of the results could also be obtained without the use of boundary triples, but we think that its use gives a nice conceptual language shortening the proofs. For the basic notion of boundary triples we refer to Section 2 .

The aim of the present article is to extend results on the relation between the standard metric and combinatorial graph Laplacian to general vertex conditions and Dirac operators. The main point here is the interpretation of Krein's Q-function as a purely combinatorial operator acting on a space given a priori by the vertex condition. To our knowledge, the interpretation of the combinatorial operator as a sort of "generalised discrete Laplacian" (see below) seems to be new, only Pankrashkin [Pa07] obtained a similarly defined combinatorial operator, but without further interpretation, in a talk held at the INI. Although the calculations for a direct proof of the spectral relation (at least for the discrete spectrum) for general vertex conditions are quite obvious, the interpretation of the resulting equations as a new type of combinatorial operator might be of its own interest.

The spectral relation between the metric and combinatorial operator in the standard case is wellknown, see for example [vB85] for the compact case and [Ca97] for the general case. Moreover, in [E97], $\delta$ and $\delta^{\prime}$ vertex conditions are considered. Dekoninck and Nicaise [DeN00] proved spectral relations for fourth order operators, and Cartwright and Woess [CW05] used integral operators on the edge.

Date: January 15, 2008.

2000 Mathematics Subject Classification. Primary 81Q10, 05C50, Secondary 34L40, 47E05, 47N50.

Key words and phrases. Quantum graphs, Laplacian, discrete graphs, spectrum. 
Although the analysis of metric graph differential operators usually reduces to a system of ODEs, it is advisable at least from a conceptional point of view not to forget the underlying global graph structure. In particular, we define an operator on the combinatorial level related to the quantum graph. Namely, we generalise the standard vertex space $\ell_{2}(V)$ on which the usual combinatorial Laplacian $\triangle$ acts as difference operator (see (3.2)) to spaces $\mathscr{G}=\bigoplus_{v} \mathscr{G}_{v}$ where $\mathscr{G}_{v}$ is a linear subspace of $\mathbb{C}^{\operatorname{deg} v}$, i.e., an element $F \in \mathscr{G}$ at a vertex $v$ is a vector $\underline{F}(v)=\left\{F_{e}(v)\right\}_{e} \in \mathscr{G}_{v}$ having as many components as adjacent edges to $v$. Note that for an element $\widetilde{F} \in \ell_{2}(V)$ of the standard vertex space, the value $\widetilde{F}(v)$ at $v$ is just a scalar. Having written the standard Laplacian as $\triangle=\mathrm{d}^{*} \mathrm{~d}$ with the coboundary operator

$$
\mathrm{d}: \ell_{2}(V) \longrightarrow \ell_{2}(E), \quad(\mathrm{d} \widetilde{F})_{e}=\widetilde{F}\left(\partial_{+} e\right)-\widetilde{F}\left(\partial_{-} e\right)
$$

("terminal minus initial vertex value"), we define the generalised combinatorial Laplacian on $\mathscr{G}$ as $\triangle_{\mathscr{G}}=\mathrm{d}_{\mathscr{G}}^{*} \mathrm{~d}_{\mathscr{G}}$, where

$$
\mathrm{d}_{\mathscr{G}}: \mathscr{G} \longrightarrow \ell_{2}(E), \quad\left(\mathrm{d}_{\mathscr{G}} F\right)_{e}=F_{e}\left(\partial_{+} e\right)-F_{e}\left(\partial_{-} e\right)
$$

For the resulting formula see Definition 3.7 below; and for more details on these generalised Laplacians and a relation on the kernel of metric and combinatorial operators we refer to [P07a].

The main observation is now, that Krein's Q-function for the boundary triple (also called Dirichlet-to-Neumann map, (operator-valued) Weyl Titchmarsh, Herglotz or Nevanlinna function) is closely related to $\triangle_{\mathscr{G}}$ for a boundary triple associated to the Laplacian and the Dirac operator on an equilateral metric graph. In particular, the abstract theory of boundary triples establishes a relation between the spectra and the resolvent of the quantum and combinatorial graph (see Theorem 5.7 for the Laplace and Theorems 6.5 and 7.1 for the Dirac operator). Moreover, using the results of [BGP06], we have a complete description of all spectral types (discrete and essential, absolutely and singular continuous, (pure) point) outside the Dirichlet spectrum $\Sigma^{\mathrm{D}}=\left\{(\pi k)^{2} \mid k=1,2, \ldots\right\}$ at least for an equilateral graph with lengths $\ell_{e}=1$ and "energy independent" vertex conditions, i.e., without Robin type conditions (see Remark 4.8 (iii)), cf. also [Pa07]. We stress that our approach covers all self-adjoint realisations of the Laplacian on a finite metric graph, but for energy dependent vertex conditions, we do not always obtain the spectral relation for the continuous and point spectral components.

The structure of this article is as follows: In the next section, we review basic notion and results on boundary triples needed for our purposes. In Section 3 we describe the combinatorial setting. Namely, we define generalised vertex spaces and the associated discrete Laplacian. In Section 4 we review the notion of a quantum graph and give a parametrisation of all self-adjoint vertex conditions adopted to our discrete setting. Section 5 is devoted to the study of the metric graph Laplacian via a suitable boundary triple, and similarly in Section 6 we study self-adjoint Dirac operators. In Section 7 we analyse a (non-self-adjoint) Dirac operator with symmetric components. Finally, Section 8 contains concluding remarks.

Acknowledgements. It is a pleasure to thank the organisers of the programme "Analysis on graphs and its applications" at the Isaac Newton Institute (INI) in Cambridge for the kind invitation and the very inspiring atmosphere there. In addition, the author would like to thank Pavel Exner, Jon Harrison, Peter Kuchment, and Konstantin Pankrashkin for helpful discussions. The author acknowledges the financial support of the Collaborative Research Center SFB 647 "Space - Time - Matter. Analytic and Geometric Structures". 


\section{Abstract Boundary triples}

The concept of boundary triples first appeared in [V63] in order to treat boundary conditions for PDE. Boundary triples allow to express boundary value problems in an purely operatortheoretic way. In this section, we briefly describe this concept, and closely follow the exposition in [BGP06]. For more details and a historical account including more references, we refer to [BGP06, DHMdS06].

In this section, we assume that $A$ is a closed operator in a Hilbert space $\mathscr{H}$ having at least one self-adjoint restriction.

Definition 2.1. We say that $\left(\mathscr{G}, \Gamma_{0}, \Gamma_{1}\right)$ is a boundary triple for $A$ if $\mathscr{G}$ is a Hilbert space, and if $\Gamma_{0}, \Gamma_{1}: \operatorname{dom} A \longrightarrow \mathscr{G}$ are two linear maps, called boundary operators, satisfying the following conditions:

$$
\begin{gathered}
\langle A f, g\rangle_{\mathscr{H}}-\langle f, A g\rangle_{\mathscr{H}}=\left\langle\Gamma_{0} f, \Gamma_{1} g\right\rangle_{\mathscr{G}}-\left\langle\Gamma_{1} f, \Gamma_{0} g\right\rangle_{\mathscr{G}}, \quad \forall f, g \in \operatorname{dom} A \\
\Gamma_{0} \stackrel{\Upsilon}{\oplus} \Gamma_{1}: \operatorname{dom} A \longrightarrow \mathscr{G} \oplus \mathscr{G}, \quad f \mapsto \Gamma_{0} f \oplus \Gamma_{1} f \quad \text { is surjective } \\
\operatorname{ker}\left(\Gamma_{0} \stackrel{\curlyvee}{\oplus} \Gamma_{1}\right)=\operatorname{ker} \Gamma_{0} \cap \operatorname{ker} \Gamma_{1} \quad \text { is dense in } \mathscr{H} .
\end{gathered}
$$

It can be shown that $\Gamma_{0}$ and $\Gamma_{1}$ are bounded maps (cf. [BGP06, Prop. 1.9]) if $\operatorname{dom} A$ is equipped with the graph norm defined by $\|f\|_{A}^{2}:=\|f\|^{2}+\|A f\|^{2}$. Moreover, denoting by $A_{0} \subset A$ the self-adjoint restriction of $A$, it follows that $A^{*} \subset A_{0}^{*}=A_{0} \subset A=A^{* *}$, i.e., that $A^{*}$ is symmetric having equal defect indices.

Lemma 2.2. Let $\left(\mathscr{G}, \Gamma_{0}, \Gamma_{1}\right)$ be a boundary triple for $A$ and set $\mathscr{N}^{z}:=\operatorname{ker}(A-z)$. Denote by $A_{0}$ the restriction of $A$ onto ker $\Gamma_{0}$, and assume that $A_{0}$ is self-adjoint in $\mathscr{H}$. Then the operator $\Gamma_{0} \Gamma_{\mathscr{N} z}: \mathscr{N}^{z} \longrightarrow \mathscr{G}$ is a topological isomorphism for $z \notin \sigma\left(A_{0}\right)$.

Its inverse, denoted by $\beta(z)$, defines a Krein $\Gamma$-field $z \mapsto \beta(z)$ associated to $\left(\mathscr{G}, \Gamma_{0}, \Gamma_{1}\right)$ and $A$, i.e.,

$$
\begin{gathered}
\beta(z): \mathscr{G} \longrightarrow \mathscr{N}^{z} \quad \text { is a topological isomorphism and } \\
\beta\left(z_{1}\right)=U\left(z_{1}, z_{2}\right) \beta\left(z_{2}\right), \quad z_{1}, z_{2} \notin \sigma\left(A_{0}\right),
\end{gathered}
$$

where $U\left(z_{1}, z_{2}\right):=\left(A_{0}-z_{2}\right)\left(A_{0}-z_{1}\right)^{-1}=1+\left(z_{1}-z_{2}\right)\left(A_{0}-z_{1}\right)^{-1}$.

For notational reasons, we denote the Krein $\Gamma$-field by $\beta$ instead of $\gamma$ (see [P07b] and Section 6, where we used $\gamma$ for another type of boundary operator).

Definition 2.3. The operator $Q(z):=\Gamma_{1} \beta(z): \mathscr{G} \longrightarrow \mathscr{G}$ defines the (canonical) Krein $Q$-function $z \mapsto Q(z)$.

The Krein Q-function fulfills

$$
Q\left(z_{1}\right)-Q\left(\bar{z}_{2}\right)^{*}=\left(z_{1}-z_{2}\right)\left(\beta\left(\bar{z}_{2}\right)\right)^{*} \beta\left(z_{1}\right) \quad z_{1}, z_{2} \notin \sigma\left(A_{0}\right) .
$$

In particular, $Q(z)$ is self-adjoint if $z$ is real.

Definition 2.4. Associated to a bounded operator $T$ in $\mathscr{G}$, we denote by $A^{T}$ the restriction of $A$ onto

$$
\operatorname{dom} A^{T}:=\left\{f \in \operatorname{dom} A \mid \Gamma_{1} f=T \Gamma_{0} f\right\} .
$$

It can be shown that $A^{T}$ is self-adjoint in $\mathscr{H}$ iff $T$ is self-adjoint in $\mathscr{G}$.

Remark 2.5. In order to parametrise all self-adjoint restrictions of $A$, one needs either a linear relation $T$ on $\mathscr{G}$ (i.e., a multi-valued linear "operator") or one has to modify the boundary triple into $\left(\widetilde{\mathscr{G}}, \widetilde{\Gamma}_{0}, \widetilde{\Gamma}_{1}\right)$ where $\widetilde{\mathscr{G}}$ is a subspace of $\mathscr{G}, \widetilde{P}$ its orthogonal projection and $\widetilde{\Gamma}_{p}:=\widetilde{P} \Gamma_{p}$. In this case, a (single-valued) operator $\widetilde{T}$ in $\widetilde{\mathscr{G}}$ is enough. Note that for the new boundary triple, $\widetilde{\beta}(z)=\beta(z) \widetilde{P}$ and $\widetilde{Q}(z)=\widetilde{P} Q(z) \widetilde{P}$ are Krein's $\Gamma$ - and Q-function, respectively, expressed in terms of the old ones (see [BGP06, Thm. 1.32]). 
One of the main results for Krein boundary triples is the following theorem (see e.g. [BGP06, Thms. 1.29, 3.3 and 3.16]):

Theorem 2.6. Let $T$ be a self-adjoint and bounded operator in $\mathscr{G}$ and $A^{T}$ the associated self-adjoint restriction as defined above.

(i) For $z \notin \sigma\left(A_{0}\right)$ we have $\operatorname{ker}\left(A^{T}-z\right)=\beta(z) \operatorname{ker}(Q(z)-T)$.

(ii) For $z \notin \sigma\left(A^{T}\right) \cup \sigma\left(A_{0}\right)$ we have $0 \notin \sigma(Q(z)-T)$ and Krein's resolvent formula

$$
\left(A_{0}-z\right)^{-1}-\left(A^{T}-z\right)^{-1}=\beta(z)(Q(z)-T)^{-1}(\beta(\bar{z}))^{*}
$$

holds.

(iii) We have the spectral relation

$$
\sigma_{\bullet}\left(A^{T}\right) \backslash \sigma\left(A_{0}\right)=\left\{z \in \mathbb{C} \backslash \sigma\left(A_{0}\right) \mid 0 \in \sigma_{\bullet}(Q(z)-T)\right\}
$$

for $\bullet \in\{\emptyset, \mathrm{pp}$, disc, ess $\}$, the whole, pure point (set of all eigenvalues), discrete and essential spectrum. Furthermore, the multiplicity of an eigenspace is preserved.

(iv) Assume that $(a, b) \cap \sigma\left(A_{0}\right)=\emptyset$, i.e., $(a, b)$ is a spectral gap for $A_{0}$. If Krein's Q-function and $T$ have the special form

$$
Q(z)-T=\frac{\triangle-m(z)}{n(z)}
$$

for a self-adjoint, bounded operator $\Delta$ on $\mathscr{G}$ and scalar functions $m, n$, analytic at least in $(\mathbb{C} \backslash \mathbb{R}) \cup(a, b)$ and $n(\lambda) \neq 0$ on $(a, b)$, then for $\lambda \in(a, b)$ we have

$$
\lambda \in \sigma_{\bullet}\left(A^{T}\right) \quad \Leftrightarrow \quad m(\lambda) \in \sigma_{\bullet}(\triangle)
$$

for all spectral types, namely, $\bullet \in\{\emptyset$, pp, disc, ess, ac, sc, p $\}$, the whole, pure point, discrete, essential, absolutely continuous, singular continuous and point spectrum $\left(\sigma_{\mathrm{p}}(A)=\overline{\sigma_{\mathrm{pp}}(A)}\right)$. Again, the multiplicity of an eigenspace is preserved.

\section{Discrete graphs and general Laplacians}

In this section, we define a generalised discrete Laplacian, which occurs in Krein's Q-function for a boundary triple associated to an equilateral metric graph. We first fix some notation for graphs.

Suppose $X$ is a discrete, weighted graph given by $(V, E, \partial, \ell)$ where $(V, E, \partial)$ is a usual graph, i.e., $V$ denotes the set of vertices, $E$ denotes the set of edges, $\partial: E \longrightarrow V \times V$ associates to each edge $e$ the pair $\left(\partial_{-} e, \partial_{+} e\right)$ of its initial and terminal point (and therefore an orientation). That $X$ is an (edge-)weighted graph means that there is a length or (inverse) edge weight function $\ell: E \longrightarrow$ $(0, \infty)$ associating to each edge $e$ a length $\ell_{e}$. For simplicity, we consider internal edges only, i.e., edges of finite length $\ell_{e}<\infty$, and we also make the following assumption on the lower bound of the edge lengths:

Assumption 3.1. Throughout this article we assume that there is a constant $\ell_{0}>0$ such that

$$
\ell_{e} \geq \ell_{0}, \quad e \in E,
$$

i.e., that the weight function $\ell^{-1}$ is bounded. Without loss of generality, we also assume that $\ell_{e} \leq 1$

For each vertex $v \in V$ we set

$$
E_{v}^{ \pm}:=\left\{e \in E \mid \partial_{ \pm} e=v\right\} \quad \text { and } \quad E_{v}:=E_{v}^{+} \bullet E_{v}^{-}
$$

i.e., $E_{v}^{ \pm}$consists of all edges starting $(-)$resp. ending $(+)$at $v$ and $E_{v}$ their disjoint union. Note that the disjoint union is necessary in order to allow self-loops, i.e., edges having the same initial and terminal point. The degree of $v \in V$ is defined as

$$
\operatorname{deg} v:=\left|E_{v}\right|=\left|E_{v}^{+}\right|+\left|E_{v}^{-}\right|
$$


i.e., the number of adjacent edges at $v$. In order to avoid trivial cases, we assume that $\operatorname{deg} v \geq 1$, i.e., no vertex is isolated.

We want to introduce a vertex space allowing us to define Laplace-like combinatorial operators motivated by general vertex conditions on quantum graphs. The usual discrete Laplacian is defined on scalar functions $F: V \longrightarrow \mathbb{C}$ on the vertices $V$, namely

$$
\triangle F(v)=-\frac{1}{\operatorname{deg} v} \sum_{e \in E_{v}}\left(F\left(v_{e}\right)-F(v)\right)
$$

where $v_{e}$ denotes the vertex on $e$ opposite to $v$. Note that $\Delta$ can be written as $\Delta=\mathrm{d}^{*} \mathrm{~d}$ with

$$
\mathrm{d}: \ell_{2}(V) \longrightarrow \ell_{2}(E), \quad(\mathrm{d} F)_{e}=F\left(\partial_{+} e\right)-F\left(\partial_{-} e\right),
$$

where $\ell_{2}(V)$ and $\ell_{2}(E)$ carry the norms defined by

$$
\|F\|_{\ell_{2}(V)}^{2}:=\sum_{v \in V}|F(v)|^{2} \operatorname{deg} v \quad \text { and } \quad\|\eta\|_{\ell_{2}(E)}^{2}:=\sum_{e \in E}\left|\eta_{e}\right|^{2} \frac{1}{\ell_{e}}
$$

and $\mathrm{d}^{*}$ denotes the adjoint with respect to the corresponding inner products. We sometimes refer to functions in $\ell_{2}(V)$ and $\ell_{2}(E)$ as 0 - and 1-forms, respectively.

We would like to carry over the above concept for the vertex space $\ell_{2}(V)$ to more general vertex spaces $\mathscr{G}$. The main motivation to do so are quantum graphs with general vertex conditions as we will see in Section 4.

Definition 3.2. Denote by $\mathscr{G}_{v}^{\max }:=\mathbb{C}^{E_{v}}$ the maximal vertex space at the vertex $v \in V$, i.e., a value $\underline{F}(v) \in \mathscr{G}_{v}^{\max }$ has $\operatorname{deg} v$ components, one for each adjacent edge. A (general) vertex space at the vertex $v$ is a linear subspace $\mathscr{G}_{v}$ of $\mathscr{G}_{v}^{\max }$. The corresponding (total) vertex spaces are

$$
\mathscr{G}^{\max }:=\bigoplus_{v \in V} \mathscr{G}_{v}^{\max } \text { and } \mathscr{G}:=\bigoplus_{v \in V} \mathscr{G}_{v}
$$

respectively. Elements of $\mathscr{G}$ are also called 0 -forms. The space $\mathscr{G}$ carries its natural Hilbert norm, namely

$$
\|F\|_{\mathscr{G}}^{2}:=\sum_{v \in V}|\underline{F}(v)|^{2}=\sum_{v \in V} \sum_{e \in E_{v}}\left|F_{e}(v)\right|^{2} .
$$

We call a general subspace $\mathscr{G}$ of $\mathscr{G}^{\text {max }}$ local iff it decomposes with respect to the maximal vertex spaces, i.e., if $\mathscr{G}=\bigoplus_{v} \mathscr{G}_{v}$ and $\mathscr{G}_{v} \leq \mathscr{G}_{v}^{\max }$.

Note that $\mathscr{G}^{\max }$ also decomposes as

$$
\mathscr{G}^{\max }=\bigoplus_{e \in E} \mathbb{C}^{\partial e}
$$

by reordering the labels via

$$
E=\bigcup_{v \in V} E_{v}^{+}=\bigcup_{v \in V} E_{v}^{-}
$$

where $\mathbb{C}^{\partial e}=\mathbb{C}^{\left\{\partial_{-} e, \partial_{+} e\right\}} \cong \mathbb{C}^{2}$. Similarly, we can consider $\ell_{2}(E)$ as

$$
\ell_{2}(E)=\bigoplus_{e \in E} \frac{1}{\ell_{e}} \mathbb{C}
$$

Associated to a vertex space is an orthogonal projection $P=\bigoplus_{v \in V} P_{v}$ in $\mathscr{G}^{\max }$, where $P_{v}$ is the orthogonal projection in $\mathscr{G}_{v}^{\max }$ onto $\mathscr{G}_{v}$. Alternatively, a vertex space is characterised by fixing an orthogonal projection $P$ in $\mathscr{G}$ which is local.

Definition 3.3. Let $\mathscr{G}=\bigoplus_{v \in V} \mathscr{G}_{v}$ be a vertex space with associated projection $P$. The dual vertex space is defined by $\mathscr{G}^{\perp}:=\mathscr{G}^{\max } \ominus \mathscr{G}$ with projection $P^{\perp}=\mathbb{1}-P$.

Example 3.4. The names of the below examples for vertex spaces will become clear in the quantum graph case. For more general cases, e.g. the magnetic Laplacian, we refer to [P07a]. 
(i) Choosing $\mathscr{G}_{v}=\mathbb{C} \mathbb{1}(v)=\mathbb{C}(1, \ldots, 1)$, we obtain the continuous or standard vertex space denoted by $\mathscr{G}_{v}^{\text {std }}$. The associated projection is

$$
P_{v}=\frac{1}{\operatorname{deg} v} \mathbb{E}
$$

where $\mathbb{E}$ denotes the square matrix of rank $\operatorname{deg} v$ where all entries equal 1 . This case corresponds to the standard discrete case mentioned before. Namely, the natural identification $F \cong \widetilde{F}$ given by $\widetilde{F}(v):=F_{e}(v)$ (the former value is independent of $e \in E_{v}$ ) gives an isometry of $\mathscr{G}^{\text {std }}=\bigoplus_{v} \mathscr{G}_{v}^{\text {std }}$ onto $\ell_{2}(V)$ since the weighted norm in $\ell_{2}(V)$ and the norm in $\mathscr{G}^{\text {std }}$ agree:

$$
\|F\|_{\mathscr{G}_{\text {std }}}^{2}=\sum_{v \in V} \sum_{e \in E_{v}}\left|F_{e}(v)\right|^{2}=\sum_{v \in V}|\widetilde{F}(v)|^{2} \operatorname{deg} v=\|\widetilde{F}\|_{\ell_{2}(V)}^{2} .
$$

(ii) We call $\mathscr{G}_{v}^{\min }:=0$ the minimal or Dirichlet vertex space, similarly, $\mathscr{G}^{\max }$ is called the maximal or Neumann vertex space. The corresponding projections are $P=0$ and $P=\mathbb{1}$.

Now, we define a generalised coboundary operator or exterior derivative associated to a vertex space. We use this exterior derivative for the definition of an associated Dirac and Laplace operator below:

Definition 3.5. Let $\mathscr{G}$ be a vertex space of the graph $X$. The exterior derivative on $\mathscr{G}$ is defined via

$$
\mathrm{d}_{\mathscr{G}}: \mathscr{G} \longrightarrow \ell_{2}(E), \quad\left(\mathrm{d}_{\mathscr{G}} F\right)_{e}:=F_{e}\left(\partial_{+} e\right)-F_{e}\left(\partial_{-} e\right),
$$

mapping 0-forms onto 1-forms.

We often drop the subscript $\mathscr{G}$ for the vertex space. A proof of the next lemma can be found in [P07a, Lem. 3.3]:

Lemma 3.6. Assume (3.1), then $\mathrm{d}$ is norm-bounded by $\sqrt{2 / \ell_{0}}$. The adjoint

$$
\mathrm{d}^{*}: \ell_{2}(E) \longrightarrow \mathscr{G}
$$

fulfills the same norm bound and is given by

$$
\left(\mathrm{d}^{*} \eta\right)(v)=P_{v}\left(\left\{\frac{1}{\ell_{e}} \widehat{\eta}_{e}(v)\right\}\right) \in \mathscr{G}_{v}
$$

where $\widehat{\eta}_{e}(v):= \pm \eta_{e}$ if $v=\partial_{ \pm}$e denotes the oriented evaluation of $\eta_{e}$ at the vertex $v$.

Definition 3.7. The discrete generalised Laplacian associated to a vertex space $\mathscr{G}$ is defined as $\triangle_{\mathscr{G}}:=\mathrm{d}_{\mathscr{G}}^{*} \mathrm{~d}_{\mathscr{G}}$, i.e.,

$$
\left(\triangle_{\mathscr{G}} F\right)(v)=P_{v}\left(\left\{\frac{1}{\ell_{e}}\left(F_{e}(v)-F_{e}\left(v_{e}\right)\right)\right\}\right)
$$

for $F \in \mathscr{G}$, where $v_{e}$ denotes the vertex on $e \in E_{v}$ opposite to $v$.

Remark 3.8.

(i) From Lemma 3.6 it follows that $\triangle_{\mathscr{G}}$ is a bounded operator on $\mathscr{G}$ with norm estimated from above by $2 / \ell_{0}$.

(ii) Note that the orientation of the edges plays no role for the "second order" operator $\triangle_{\mathscr{G}}$.

(iii) We can also define a Laplacian $\triangle_{\mathscr{G}}^{1}:=\mathrm{d}_{\mathscr{G}} \mathrm{d}_{\mathscr{G}}^{*}$ acting on the space of "1-forms" $\ell_{2}(E)$. For more details and the related supersymmetric setting, we refer to [P07a]. In particular, in the equilateral case $\ell_{e}=1, \sigma\left(\Delta_{\mathscr{G}}\right) \subseteq[0,2]$, and the supersymmetric setting can be used to show the spectral relation

$$
\sigma\left(\triangle_{\mathscr{G} \perp}\right) \backslash\{0,2\}=2-\left(\sigma\left(\triangle_{\mathscr{G}}\right) \backslash\{0,2\}\right),
$$

i.e., if $\lambda \notin\{0,2\}$, then $\lambda \in \sigma\left(\triangle_{\mathscr{G} \perp}\right)$ iff $2-\lambda \in \sigma\left(\triangle_{\mathscr{G}}\right)$ (cf. [P07a, Lem. 3.13]).

\section{Example 3.9.}


(i) For the standard vertex space $\mathscr{G}^{\text {std }}$, it is convenient to use the unitary transformation from $\mathscr{G}^{\text {std }}$ onto $\ell_{2}(V)$ associating to $F \in \mathscr{G}$ the (common value) $\widetilde{F}(v):=F_{e}(v)$ as in Example 3.4 (i). Then the exterior derivative and its adjoint are unitarily equivalent to

$$
\widetilde{\mathrm{d}}: \ell_{2}(V) \longrightarrow \ell_{2}(E), \quad(\widetilde{\mathrm{d}} \widetilde{F})_{e}=\widetilde{F}\left(\partial_{+} e\right)-\widetilde{F}\left(\partial_{-} e\right)
$$

and

$$
\left(\widetilde{\mathrm{d}^{*}} \eta\right)(v)=\frac{1}{\operatorname{deg} v} \sum_{e \in E_{v}} \frac{1}{\ell_{e}} \widehat{\eta}_{e}(v),
$$

i.e., $\widetilde{d}$ is the classical coboundary operator and $\widetilde{d}^{*}$ its adjoint.

Moreover, the corresponding discrete Laplacian $\triangle_{\mathscr{C g s t d}}$ is unitarily equivalent to the usual discrete Laplacian $\Delta=\widetilde{\mathrm{d}}^{*} \widetilde{\mathrm{d}}$ defined in (3.2) as one can easily check.

(ii) For the minimal vertex space $\mathscr{G}^{\min }=0$, we have $\mathrm{d}=0, \mathrm{~d}^{*}=0$ and $\Delta_{\mathscr{G} \min }=0$. Obviously, these operators are decoupled, i.e., they do not feel any connection information of the graph.

(iii) For the maximal vertex space, we have (denoting $d=d^{\max }$ )

$$
\left(\mathrm{d}^{*} \eta\right)_{e}(v)=\frac{1}{\ell_{e}} \widehat{\vartheta}_{e}(v)
$$

The operator $\mathrm{d}=\mathrm{d}^{\text {max }}$ decomposes as $\bigoplus_{e} \mathrm{~d}_{e}$ with respect to the decomposition of $\mathscr{G}^{\max }$ in Eq. (3.3) and $\ell_{2}(E)$ in Eq. (3.5). In particular,

$$
\left(\mathrm{d}_{e}: \mathbb{C}^{\partial e} \longrightarrow \mathbb{C}\right) \cong\left(\begin{array}{ll}
-1 & 1
\end{array}\right) \text { and }\left(\mathrm{d}_{e}^{*}: \mathbb{C} \longrightarrow \mathbb{C}^{\partial e}\right) \cong \frac{1}{\ell_{e}}\left(\begin{array}{c}
-1 \\
1
\end{array}\right)
$$

where $F_{e}=\left(F_{e}\left(\partial_{-} e\right), F_{e}\left(\partial_{+} e\right)\right) \in \mathbb{C}^{\partial e}$. The corresponding Laplacian is given by

$$
\left(\triangle_{\mathscr{G} \max } F\right)_{e}(v)=\left\{\frac{1}{\ell_{e}}\left(F_{e}(v)-F_{e}\left(v_{e}\right)\right)\right\}_{e \in E_{v}}
$$

and this operator decomposes as $\bigoplus_{e}\left(\triangle_{\mathscr{G} \max }\right)_{e}$ with respect to the decomposition of $\mathscr{G}^{\max }$ in Eq. (3.3), where

$$
\left(\left(\Delta_{\mathscr{G} \max }\right)_{e}: \mathbb{C}^{\partial e} \longrightarrow \mathbb{C}^{\partial e}\right) \cong \frac{1}{\ell_{e}}\left(\begin{array}{cc}
1 & -1 \\
-1 & 1
\end{array}\right) .
$$

Again, the operators are decoupled. In particular, any connection information of the graph is lost.

\section{QuAntum GRAPhS}

In this section, we briefly review the notion of a metric graph and differential operators acting on it.

Definition 4.1. A (continuous) metric graph $X=(V, E, \partial, \ell)$ is formally given by the same data as a discrete (edge-)weighted graph. The difference is the interpretation of the space $X$ : We define $X$ as

$$
X:=\bigcup_{e \in E} I_{e} / \sim_{\psi}
$$

where $I_{e}:=\left[0, \ell_{e}\right]$ and where we identify $x \sim_{\psi} y$ iff $\psi(x)=\psi(y)$ with

$$
\psi: \bigcup_{e \in E}\left\{0, \ell_{e}\right\} \longrightarrow V, \quad 0_{e} \mapsto \partial_{-} e, \quad \ell_{e} \mapsto \partial_{+} e .
$$


In the sequel, we often drop the edge subscript $e$, e.g., we use $x=x_{e}$ as coordinate and denote by $\mathrm{d} x=\mathrm{d} x_{e}$ the Lebesgue measure on $I_{e}$. In this way, the space $X$ becomes a metric measure space by defining the distance between two points to be the length of the shortest path in $X$ joining these points.

We now define several Sobolev spaces associated with $X$. Our basic Hilbert space is

$$
\mathrm{L}_{2}(X):=\bigoplus_{e \in E} \mathrm{~L}_{2}\left(I_{e}\right)
$$

with its natural norm defined by

$$
\|f\|_{\mathrm{L}_{2}(X)}^{2}:=\sum_{e} \int_{I_{e}}\left|f_{e}(x)\right|^{2} \mathrm{~d} x .
$$

For this norm, we often omit the label indicating the space, i.e., we write $\|f\|=\|f\|_{\mathrm{L}_{2}(X)}$. More generally, the decoupled or maximal Sobolev space of order $k$ is

$$
\mathrm{H}_{\max }^{k}(X):=\bigoplus_{e \in E} \mathrm{H}^{k}\left(I_{e}\right)
$$

with norm defined by

$$
\|f\|_{\mathrm{H}_{\max }^{k}(X)}^{2}:=\sum_{e}\left\|f_{e}\right\|_{\mathrm{H}^{k}\left(I_{e}\right)}^{2}=\sum_{e} \int_{I_{e}}\left(\left|f_{e}(x)\right|^{2}+\left|f_{e}^{\prime}(x)\right|^{2}+\cdots+\left|f_{e}^{(k)}(x)\right|^{2}\right) \mathrm{d} x .
$$

Obviously, for $k=0$, there is no difference between $\mathrm{L}_{2}(X)$ and the decoupled space. Namely, the evaluation of a function at a point only makes sense if $k \geq 1$ due to Lemma 4.3.

We will now define the vertex evaluation maps. The reason for two different types of evaluations at a vertex is the simple form of the integration by parts formula on a metric graph in Lemma 4.4 below.

Definition 4.2. For $f \in \mathrm{H}_{\max }^{1}(X)$, we denote

$$
\underline{f}=\{\underline{f}(v)\}_{v \in V}, \quad \underline{f}(v)=\left\{f_{e}(v)\right\}_{e \in E_{v}}, \quad f_{e}(v):= \begin{cases}f_{e}(0), & v=\partial_{-} e \\ f_{e}\left(\ell_{e}\right), & v=\partial_{+} e\end{cases}
$$

the unoriented evaluation at the vertex $v$. Similarly, for $g \in \mathrm{H}_{\max }^{1}(X)$, we denote

$$
\underline{\widehat{g}}=\{\underline{g}(v)\}_{v \in V}, \quad \underline{g}(v)=\left\{\widehat{g}_{e}(v)\right\}_{e \in E_{v}}, \quad \widehat{g}_{e}(v):= \begin{cases}-g_{e}(0), & v=\partial_{-} e \\ g_{e}\left(\ell_{e}\right), & v=\partial_{+} e\end{cases}
$$

the oriented evaluation at the vertex $v$.

The following lemma is a simple consequence of a standard estimate for Sobolev spaces (see e.g. [P07a, Lem. 5.2]):

Lemma 4.3. Assume the condition (3.1) on the edge lengths, i.e., there is $\ell_{0} \in(0,1]$ such that $\ell_{e} \geq \ell_{0}$ for all $e \in E$. Then the evaluation maps

$$
\text { •.: } \mathrm{H}_{\max }^{1}(X) \longrightarrow \mathscr{G}^{\max }, \quad f \mapsto \underline{f} \quad \text { and } \underline{\boldsymbol{0}}: \mathrm{H}_{\max }^{1}(X) \longrightarrow \mathscr{G}^{\max }, \quad g \mapsto \underline{g},
$$

are bounded by $2 / \sqrt{\ell_{0}}$.

For a general vertex space $\mathscr{G}$, i.e., a closed subspace of $\mathscr{G}^{\max }:=\bigoplus_{v \in V} \mathbb{C}^{E_{v}}$, we set

$$
\mathrm{H}_{\mathscr{G}}^{1}(X):=\left\{f \in \mathrm{H}_{\max }^{1}(X) \mid \underline{f} \in \mathscr{G}\right\}=(\underline{\bullet})^{-\mathscr{G}},
$$

i.e., the pre-image of $\mathscr{G}$ under the (unoriented) evaluation map, and similarly,

$$
\mathrm{H}_{\hat{\mathscr{G}}}^{1}(X):=\left\{g \in \mathrm{H}_{\max }^{1}(X) \mid \underline{g} \in \mathscr{G}\right\}=(\underline{\bullet})^{-\mathscr{G}}
$$

the pre-image of $\mathscr{G}$ under the (oriented) evaluation map. In particular, both spaces are closed in $\mathrm{H}_{\max }^{1}(X)$ and therefore themselves Hilbert spaces. 
We can now show the integration by parts formula on a metric graph:

Lemma 4.4. For $f, g \in \mathrm{H}_{\max }^{1}(X)$, we have

$$
\left\langle f^{\prime}, g\right\rangle=\left\langle f,-g^{\prime}\right\rangle+\langle\underline{f}, \underline{g}\rangle_{\mathscr{G} \max } .
$$

Proof. Integration by parts yields

$$
\begin{aligned}
\left\langle f^{\prime}, g\right\rangle+\left\langle f, g^{\prime}\right\rangle=\sum_{e \in E}\left(\left\langle f^{\prime}, g\right\rangle_{\mathrm{L}_{2}\left(I_{e}\right)}+\left\langle f, g^{\prime}\right\rangle_{\mathrm{L}_{2}\left(I_{e}\right)}\right)=\sum_{e \in E}\left[(\bar{f} g)_{e}\left(\partial_{+} e\right)-(\bar{f} g)_{e}\left(\partial_{-} e\right)\right] \\
=\sum_{v \in V} \sum_{e \in E_{v}} \bar{f}_{e}(v) \widehat{g}_{e}(v)=\sum_{v \in V}\langle\underline{f}(v), \underline{g}(v)\rangle_{\mathbb{C}^{E_{v}}}=\langle\underline{f}, \underline{\hat{g}}\rangle_{\mathscr{G} \max }
\end{aligned}
$$

reordering the labels with (3.4). Note that the evaluation is well-defined due to Lemma 4.3.

If we fix the function $f$ to have vertex values in $\mathscr{G}$, we obtain:

Corollary 4.5. For $f \in \mathrm{H}_{\mathscr{G}}^{1}(X), g \in \mathrm{H}_{\max }^{1}(X)$, we have

$$
\left\langle f^{\prime}, g\right\rangle=\left\langle f,-g^{\prime}\right\rangle+\langle\underline{f}, P \underline{g}\rangle_{\mathscr{G}} .
$$

Proof. The formula follows immediately from

$$
\langle\underline{f}, \underline{\hat{g}}\rangle_{\mathscr{G}_{\max }}=\langle P \underline{f}, \underline{\hat{g}}\rangle_{\mathscr{G} \max }=\langle\underline{f}, P \underline{\hat{g}}\rangle_{\mathscr{G}}
$$

since $\underline{f} \in \mathscr{G}$, i.e., $\underline{f}=P \underline{f}$.

Following the notation in [K04], we make the following definition:

Definition 4.6. A quantum graph $X$ is a metric graph together with a self-adjoint differential operator.

In the case of a Laplace operator on a metric graph, i.e., an operator $\Delta_{X}$ acting as $\left(\Delta_{X} f\right)_{e}=-f_{e}^{\prime \prime}$ on each edge $e \in E$, we have the following characterisation from [K04, Thm. 17]:

Theorem 4.7. Assume the lower bound on the edge lengths (3.1), namely $\ell_{e} \geq \ell_{0}>0$. Let $\mathscr{G} \leq$ $\mathscr{G}^{\max }$ be a (closed) vertex space with orthogonal projection $P$, and let $L$ be a self-adjoint, bounded operator on $\mathscr{G}$. Then the Laplacian $\Delta_{(\mathscr{G}, L)}$ with domain

is self-adjoint.

$$
\operatorname{dom} \Delta_{(\mathscr{G}, L)}:=\left\{f \in \mathrm{H}_{\max }^{2}(X) \mid \underline{f} \in \mathscr{G}, \quad{\stackrel{P}{f^{\prime}}}^{\prime}=L \underline{f}\right\}
$$

\section{Remark 4.8.}

(i) For finite graphs, the converse statement is true, i.e., if $\Delta$ is a self-adjoint Laplacian then $\Delta=\Delta_{(\mathscr{G}, L)}$ for some vertex space $\mathscr{G}$ and a bounded operator $L$ (not necessarily local). In particular, for finite graphs, our parametrisation by $\mathscr{G}$ and $L$ covers all self-adjoint realisations of Laplacians on the metric graph. Note that the theorem and its converse (see Remark 5.6) also follow from the boundary space setting developped in the next section, namely $\Delta_{(\mathscr{G}, L)}=\Delta^{L}$, where the latter notation was given in Definition 2.4.

For infinite graphs, the operator $L$ may become unbounded but we do not consider this case here.

(ii) If we use the further decomposition of $\mathscr{G}$ into $\mathscr{G}_{0}:=\operatorname{ker} L$ and $\mathscr{G}_{1}:=\mathscr{G} \ominus \mathscr{G}_{0}$ with associated orthogonal projections $P_{0}$ and $P_{1}$, then $L_{1}:=L \uparrow_{\mathscr{G}_{1}}$ is invertible, and $\left.f \in \operatorname{dom} \Delta_{(\mathscr{G}, L)}\right)$ iff $f \in \mathrm{H}_{\max }^{2}(X)$ and

$$
P^{\perp} \underline{f}=0, \quad P_{0} \tilde{f}^{\prime}=0, \quad P_{1} \tilde{f}^{\prime}=L_{1} P_{1} \underline{f},
$$

i.e., the vertex condition splits into a Dirichlet, Neumann and Robin part (cf. [FKW07, Thm. 2]). The self-adjoint Laplacian is therefore described by the decomposition $\mathscr{G}^{\max }=$ $\mathscr{G}_{0} \oplus \mathscr{G}_{1} \oplus \mathscr{G}^{\perp}$ and an invertible, bounded operator $L_{1}$ on $\mathscr{G}_{1}$. 
(iii) In [KoS99] (see also [KoPS07] and [Ha00]) there is another way of parametrising all selfadjoint vertex conditions, namely for bounded operators $A, B$ on $\mathscr{G}^{\text {max }}$,

$$
\operatorname{dom} \Delta_{(A, B)}=\left\{f \in \mathrm{H}_{\max }^{2}(X) \mid A \underline{f}=B \underline{f}^{\prime}\right\}
$$

is the domain of a self-adjoint operator $\Delta_{(A, B)}$ iff

(a) $A \stackrel{\hat{\oplus}}{ } B: \mathscr{G}^{\max } \oplus \mathscr{G}^{\max } \longrightarrow \mathscr{G}^{\max }, F \oplus G \mapsto A F+B G$, is surjective

(b) $A B^{*}$ is self-adjoint, i.e., $A B^{*}=B A^{*}$.

Given a vertex space $\mathscr{G} \leq \mathscr{G}^{\max }$ and a bounded operator $L$ on $\mathscr{G}$, we have $\Delta_{(A, B)}=\Delta_{(\mathscr{G}, L)}$ if we choose

$$
A \cong\left(\begin{array}{ll}
L & 0 \\
0 & \mathbb{1}
\end{array}\right) \text { and } B=P \cong\left(\begin{array}{ll}
\mathbb{1} & 0 \\
0 & 0
\end{array}\right)
$$

with respect to the decomposition $\mathscr{G}^{\max }=\mathscr{G} \oplus \mathscr{G}^{\perp}$. The associated scattering matrix with spectral parameter $\mu=\sqrt{\lambda}$ is

$$
S(\mu):=-(A+\mathrm{i} \mu B)^{-1}(A-\mathrm{i} \mu B) \cong\left(\begin{array}{cc}
-(L+\mathrm{i} \mu \mathbb{1})^{-1}(L-\mathrm{i} \mu \mathbb{1}) & 0 \\
0 & -\mathbb{1}
\end{array}\right) .
$$

In particular, $S(\mu)$ is independent of $\mu$ iff $L=0$, and in this case, we have $S(\mu)=\mathbb{1} \oplus-\mathbb{1}$ for all $\mu$. Therefore, we call the vertex conditions parametrised by $(\mathscr{G}, 0)$ energy independent. For an equivalent characterisation we refer to [KoPS07, Prop. 2.4].

\section{METRIC GRAPH LAPLACIANS AND BOUNDARY TRIPLES}

We now apply the concept of a boundary triple to a quantum graph $X$ with vertex boundary space $\mathscr{G}$ and projection $P$ onto $\mathscr{G}$ in $\mathscr{G}^{\max }$. Our Hilbert space will be $\mathscr{H}:=\mathrm{L}_{2}(X)$ and we define the (generally non-self-adjoint) Laplacian $\Delta$ on the domain

$$
\operatorname{dom} \Delta:=\mathrm{H}_{\mathscr{G}}^{2}(X):=\left\{f \in \mathrm{H}_{\max }^{2}(X) \mid \underline{f} \in \mathscr{G}\right\},
$$

i.e., we fix the vertex values $\underline{f}$ to be in the vertex space $\mathscr{G}$.

We first can show the following estimate:

Lemma 5.1. Under the assumption (3.1) there is a constant $C=C\left(\ell_{0}\right)$ such that

$$
\left\|f^{\prime}\right\|^{2} \leq C\left(\|f\|^{2}+\left\|f^{\prime \prime}\right\|^{2}\right)
$$

for all $f \in \mathrm{H}_{\max }^{2}(X)$.

Proof. The above estimate for the whole graph follows easily from the corresponding estimate on each interval $I_{e}$. But for an interval of positive length $\ell_{e} \geq \ell_{0}$, the estimate on $I_{e}$ follows from basic Sobolev theory and the constant depends only on $\ell_{0}$ (for a similar proof, see e.g. [HP06, Lem. C.4]).

Corollary 5.2. Under the assumption (3.1) the operator $\Delta=\Delta_{\mathscr{G}}^{\max }$ with domain $\operatorname{dom} \Delta=\mathrm{H}_{\mathscr{G}}^{2}(X)$ is closed.

Proof. Due to the estimate in Lemma 5.1, the Sobolev and the graph norms given by

$$
\|f\|_{\mathrm{H}_{\max }^{2}(X)}^{2}:=\|f\|^{2}+\left\|f^{\prime}\right\|^{2}+\left\|f^{\prime \prime}\right\|^{2} \text { and }\|f\|_{\Delta}^{2}:=\|f\|^{2}+\left\|f^{\prime \prime}\right\|^{2} \text {, }
$$

respectively, are equivalent. Since $\mathrm{H}_{\mathscr{G}}^{2}(X)$ is a closed subspace in $\mathrm{H}_{\text {max }}^{2}(X)$ (the pre-image of the closed space $\mathscr{G} \leq \mathscr{G}^{\max }$ under the bounded map $\left.\mathrm{H}_{\max }^{2}(X) \hookrightarrow \mathrm{H}_{\max }^{1}(X) \stackrel{(\bullet)}{\rightarrow} \mathscr{G}^{\max }\right), \mathrm{H}_{\mathscr{G}}^{2}(X)$ is complete in the Sobolev norm and therefore also in the graph norm, i.e., $\Delta$ is closed on $\mathrm{H}_{\mathscr{G}}^{2}(X)$.

We define the boundary operators by

$$
\begin{aligned}
\Gamma_{0}: \operatorname{dom} \Delta \longrightarrow \mathscr{G}, & f \mapsto \underline{f} \\
\Gamma_{1}: \operatorname{dom} \Delta \longrightarrow \mathscr{G}, & f \mapsto P \underline{f}^{\prime}
\end{aligned}
$$


(cf. Lemma 4.3 for the definition of the evaluation maps).

Lemma 5.3. Under the assumption (3.1) and with the above notation, $\left(\mathscr{G}, \Gamma_{0}, \Gamma_{1}\right)$ is a boundary triple for the Laplacian $\Delta$ on $\operatorname{dom} \Delta=\mathrm{H}_{\mathscr{G}}^{2}(X)$.

Proof. In order to show Green's formula (2.1a), we check that

$$
\langle\Delta f, g\rangle-\langle f, \Delta g\rangle=-\left\langle P \underline{f}^{\prime}, \underline{g}\right\rangle_{\mathscr{G}}+\left\langle\underline{f}, P \underline{g}^{\prime}\right\rangle_{\mathscr{G}}=\left\langle\Gamma_{0} f, \Gamma_{1} g\right\rangle_{\mathscr{G}}-\left\langle\Gamma_{1} f, \Gamma_{0} g\right\rangle_{\mathscr{G}}
$$

using Corollary 4.5.

For the surjectivity (2.1b) one has to construct a function $f \in \mathrm{H}_{\mathscr{G}}^{2}(X)$ with prescribed values $\underline{f}=F$ and $\underline{f}^{\prime}=G$ for given $F, G \in \mathscr{G}$. Clearly, this can be done locally at each vertex for a function vanishing at points with distance more than $\ell_{0} / 2$ from each vertex. The global lower bound on each length $\ell_{e} \geq \ell_{0}$ assures that the different parts of the functions near each vertex have disjoint supports and that the summability of $F$ and $G$ (i.e., $F, G \in \mathscr{G}$ ) implies the integrability of $f, f^{\prime}$ and $f^{\prime \prime}$ on $X$ for an appropriate choice of $f$. The density condition (2.1c) follows from the density of the space of smooth functions with compact support away from the vertices.

In order that $\Delta$ has self-adjoint restrictions we need to ensure that $\Delta$ has at least one. The natural candidate is the restriction $\Delta_{0}$ of $\Delta$ to $\operatorname{ker} \Gamma_{0}$. Since

$$
\Delta_{0}=\bigoplus_{e \in E} \Delta_{I_{e}}^{\mathrm{D}}
$$

where $\Delta_{I_{e}}^{\mathrm{D}}$ denotes the Laplacian on $I_{e}$ with Dirichlet boundary conditions, it follows that $\Delta_{0}$ is self-adjoint. Moreover, the spectrum of $\Delta_{0}$ is the union of the individual Dirichlet spectra $\sigma\left(\Delta_{I_{e}}^{\mathrm{D}}\right)=\left\{\left(\pi k / \ell_{e}\right)^{2} \mid k=1,2, \ldots\right\}$.

Lemma 5.4. The Krein $\Gamma$-field $z \mapsto\left(\beta(z): \mathscr{G} \longrightarrow \mathscr{N}^{z}=\operatorname{ker}(\Delta-z)\right)$ associated to the boundary triple $\left(\mathscr{G}, \Gamma_{0}, \Gamma_{1}\right)$ is given by $f=\gamma(z) F$ with

$$
f_{e}(x)=F_{e}\left(\partial_{-} e\right) s_{-, e, z}(x)+F_{e}\left(\partial_{+} e\right) s_{+, e, z}(x),
$$

where $^{1}$

for $z \notin \sigma\left(\Delta_{0}\right)$.

$$
s_{-, e, z}(x)=\frac{\sin \left(\sqrt{z}\left(\ell_{e}-x\right)\right)}{\sin \sqrt{z} \ell_{e}} \quad \text { and } \quad s_{+, e, z}(x)=\frac{\sin (\sqrt{z} x)}{\sin \sqrt{z} \ell_{e}} .
$$

Proof. Clearly, the fundamental solutions $s_{ \pm, e, z}$ solve the eigenvalue equation on each edge. Furthermore, $f_{e}(v)=F_{e}(v)$ for $v=\partial_{ \pm}$e, i.e., $\beta(z) \Gamma_{0} f=f$ for $f \in \mathscr{N}^{z}$ and $\Gamma_{0} \beta(z) F=F$ and the assertion follows.

The proof of the following lemma is a straightforward calculation from the definition $Q(z):=$ $\Gamma_{1} \beta(z)$ of the (canonical) Krein Q-function:

Lemma 5.5. The Krein Q-function $z \mapsto(Q(z): \mathscr{G} \longrightarrow \mathscr{G}), z \notin \sigma\left(\Delta_{0}\right)$, associated to the boundary triple $\left(\mathscr{G}, \Gamma_{0}, \Gamma_{1}\right)$ for $\Delta$ is given by

$$
(Q(z) F)(v)=P_{v}\left\{\frac{\sqrt{z}}{\sin \left(\sqrt{z} \ell_{e}\right)}\left[\cos \left(\sqrt{z} \ell_{e}\right) F_{e}(v)-F_{e}\left(v_{e}\right)\right]\right\}_{e \in E_{v}} .
$$

In particular, if the metric graph is equilateral (without loss of generality, $\ell_{e}=1$ ), we have

where

$$
Q(z)=\frac{1}{\sin _{1} \sqrt{z}}\left[\Delta_{\mathscr{G}}-(1-\cos \sqrt{z})\right]=\frac{1}{\sin _{1} \sqrt{z}} \Delta_{\mathscr{G}}-\left(\sqrt{z} \tan \frac{\sqrt{z}}{2}\right),
$$

$$
\sin _{1} w:=\frac{\sin w}{w}
$$

and its canonical analytic continuation $\sin _{1} 0:=1$.

\footnotetext{
${ }^{1}$ For $z=0$, we set $s_{-, e, 0}(x):=1-x / \ell_{e}$ and $s_{+, e, 0}(x):=x / \ell_{e}$.
} 
For a vertex space $\mathscr{G}$ and a bounded, self-adjoint operator $L$ on $\mathscr{G}$, we obtain a self-adjoint Laplacian $\Delta_{(\mathscr{G}, L)}$ with domain

$$
\operatorname{dom} \Delta_{(\mathscr{G}, L)}:=\left\{f \in \mathrm{H}_{\max }^{2}(X) \mid \underline{f} \in \mathscr{G}, \quad \stackrel{P^{\prime}}{f^{\prime}}=L \underline{f}\right\} .
$$

Note that $\Delta_{(\mathscr{G}, L)}=\Delta^{L}$ where $\Delta^{L}$ is defined in Definition 2.4 for the boundary triple $\left(\mathscr{G}, \Gamma_{0}, \Gamma_{1}\right)$ and the operator $\Delta$ with domain $\mathrm{H}_{\mathscr{G}}^{2}(X)$. For an equilateral graph with $\ell_{e}=1$, the operator $Q(z)-L$ has the special form

$$
Q(z)-L=\frac{\triangle_{\mathscr{G}}-(1-\cos \sqrt{z})-\left(\sin _{1} \sqrt{z}\right) L}{\sin _{1} \sqrt{z}} .
$$

Remark 5.6. Note that the parametrisation $(\mathscr{G}, L)$ covers already all self-adjoint realisations of the Laplacian: In Remark 2.5 we have seen that instead of a linear relation needed for $L$, one might also change the boundary triple into $\left(\widetilde{\mathscr{G}}, \widetilde{\Gamma}_{0}, \Gamma_{1}\right)$ with $\widetilde{\mathscr{G}} \leq \mathscr{G}$ and projection $\widetilde{P}, \widetilde{\Gamma} p:=\widetilde{P} \Gamma_{p}$; now a (single-valued) operator $\widetilde{L}$ in $\widetilde{\mathscr{G}}$ is enough. Note that we only have to replace the vertex space $\mathscr{G}$ by the new one $\widetilde{\mathscr{G}}$ : For example, the new Q-function $\widetilde{Q}(z)=\widetilde{P} Q(z) \widetilde{P}$ contains the generalised discrete Laplacian $\Delta_{\widetilde{\mathscr{G}}}$ for the new vertex space $\widetilde{\mathscr{G}}$ since $\Delta_{\widetilde{\mathscr{G}}}=\widetilde{P} \triangle_{\mathscr{G}} \widetilde{P}$.

Theorem 2.6 yields in this situation:

Theorem 5.7. Assume the lower bound on the edge lengths (3.1).

(i) For $z \notin \sigma\left(\Delta_{0}\right)$ we have the explicit formula for the eigenspaces

$$
\operatorname{ker}\left(\Delta_{(\mathscr{G}, L)}-z\right)=\beta(z) \operatorname{ker}(Q(z)-L) .
$$

(ii) For $z \notin \sigma\left(\Delta_{(\mathscr{G}, L)}\right) \cup \sigma\left(\Delta_{0}\right)$ we have $0 \notin \sigma(Q(z)-L)$ and Krein's resolvent formula

$$
\left(\Delta_{(\mathscr{G}, L)}-z\right)^{-1}=\left(\Delta_{0}-z\right)^{-1}-\beta(z)(Q(z)-L)^{-1}(\beta(\bar{z}))^{*}
$$

holds.

(iii) We have the spectral relation

$$
\sigma_{\bullet}\left(\Delta_{(\mathscr{G}, L)}\right) \backslash \sigma\left(\Delta_{0}\right)=\left\{\lambda \in \mathbb{C} \backslash \sigma\left(\Delta_{0}\right) \mid 0 \in \sigma_{\bullet}(Q(\lambda)-L)\right\} .
$$

In particular, for an equilateral graph (i.e., $\ell_{e}=1$ ), we have

$$
\lambda \in \sigma_{\bullet}\left(\Delta_{(\mathscr{G}, L)}\right) \Leftrightarrow 0 \in \sigma_{\bullet}\left(\Delta_{\mathscr{G}}-\left(\sin _{1} \sqrt{\lambda}\right) L-(1-\cos \sqrt{\lambda})\right)
$$

for $\lambda \notin \Sigma^{\mathrm{D}}=\left\{(\pi k)^{2} \mid k=1,2, \ldots\right\}$, where $\Delta_{\mathscr{G}}$ is the discrete Laplacian associated to the vertex space $\mathscr{G}$ (see Definition 3.7) and where $\bullet \in\{\emptyset$, pp, disc, ess $\}$. Furthermore, the multiplicity of an eigenspace is preserved.

(iv) Assume that the graph is equilateral, and additionally, that $L=L_{0}$ id for some constant $L_{0} \in \mathbb{R}$, then for $\lambda$ in the spectral gap $\left(\pi^{2} k^{2}, \pi^{2}(k+1)^{2}\right)(k=1,2, \ldots)$ of $\Delta_{0}$ or $\lambda<\pi^{2}$, we have

$$
\lambda \in \sigma_{\bullet}\left(\Delta_{(\mathscr{G}, L)}\right) \Leftrightarrow\left(\sin _{1} \sqrt{\lambda}\right) L_{0}+(1-\cos \sqrt{\lambda}) \in \sigma_{\bullet}\left(\Delta_{\mathscr{G}}\right)
$$

for all spectral types, namely, $\bullet \in\{\emptyset$, pp, disc, ess, ac, sc, p $\}$. Again, the multiplicity of an eigenspace is preserved. 
Remark 5.8.

(i) The above result extends the analysis done in [Pa06] (see also [E97, Ca97] and the references in these articles) for the standard vertex space $\mathscr{G}^{\text {std }}$ to all types of self-adjoint vertex conditions parametrised by $\mathscr{G}$ and $L$. In [BGP06, BGP07], also magnetic Laplacians are considered. Note that a magnetic Laplacian can also be understood as generalised Laplacian for a suitable vertex space (cf. [P07a, Rems. 2.10 (vii) and 2.11]). The spectral relation was already announced in [Pa07] also for general vertex conditions.

(ii) The eigenspaces in (i) for an equilateral graph with $L=0$ can be constructed from the discrete data $F \in \operatorname{ker}\left(\triangle_{\mathscr{G}}-(1-\cos \sqrt{z})\right)$ by applying Krein's $\Gamma$-function, the "solution operator", namely, $f=\beta(z) F$ is the corresponding eigenfunction of the metric graph Laplacian. The converse is also true: Given $f \in \operatorname{ker}\left(\Delta_{(\mathscr{G}, 0)}-z\right)$, then the corresponding eigenfunction $F \in \operatorname{ker}\left(\triangle_{\mathscr{G}}-(1-\cos \sqrt{z})\right)$ is just the restriction of $f$ to the vertices, namely $F=f$.

(iii) The resolvent formula in Theorem 5.7 (ii) is very explicit, since

$$
\left(\Delta_{0}-z\right)^{-1}=\bigoplus_{e \in E}\left(\Delta_{I_{e}}^{\mathrm{D}}-z\right)^{-1}
$$

is decoupled and explicit formulas for the resolvent on the interval are known. Furthermore, in the equilateral case and if $L=0$, the second term on the RHS in (ii) contains the resolvent of $\Delta_{\mathscr{G}}$, namely,

$$
Q(z)^{-1}=\sin _{1} \sqrt{z}\left(\Delta_{\mathscr{G}}-(1-\cos \sqrt{z})\right)^{-1} .
$$

In particular, the analysis of the metric graph resolvent is reduced to the analysis of the discrete Laplacian resolvent (see also [KoS06, KoPS07]).

Krein's resolvent formula (ii) is very useful when analysing further properties of the quantum graph $\left(X, \Delta_{(\mathscr{G}, L)}\right.$ via the resolvent.

(iv) For simplicity, we do not consider the exceptional Dirichlet spectrum here. One needs more information of the graph in order to decide whether these exceptional values are in the spectrum of the metric graph operator or not (see e.g. [Ca97]).

(v) Theorem 5.7 (iii) can be used to show the existence of spectral gaps for the metric graph Laplacian. For example, $\Delta_{(\mathscr{G}, 0)}$ has spectral gaps iff $\sigma\left(\triangle_{\mathscr{G}}\right) \neq[0,2]$. On a periodic graph, i.e., an Abelian covering $\tilde{X} \rightarrow X$ with finite graph $X$, both operators can be analysed using Floquet theory, but the spectral problem on the vertex space is reduced to a family of discrete Laplacians acting on a finite-dimensional space (see for example the results on carbon nano-structures [KP07]).

(vi) For "fractal" metric graphs, i.e., metric graphs, where $\inf _{e} \ell_{e}=0$, the corresponding discrete Laplacian $\triangle_{\mathscr{G}}$ is unbounded, and one cannot use the standard boundary triple theory. In this situation we refer to the first order approach in [P07b] developped originally for the PDE case.

\section{Self-Adjoint Dirac operators}

In this section, we discuss Dirac type operators on the metric graph $X$. In particular, for $m \in \mathbb{R}$, we consider a differential operator acting formally as

$$
D_{e}=-\mathrm{i} \partial_{x_{e}} \otimes\left(\begin{array}{cc}
0 & -\mathrm{i} \\
\mathrm{i} & 0
\end{array}\right)+m \otimes\left(\begin{array}{cc}
1 & 0 \\
0 & -1
\end{array}\right)=\left(\begin{array}{cc}
m & -\partial_{x_{e}} \\
\partial_{x_{e}} & -m
\end{array}\right)
$$

on $\mathbb{C}^{2}$-valued functions on the interval $I_{e}$ and describe self-adjoint realisations of this differential expression on the metric graph.

We fix a vertex space $\mathscr{G}$ and define

$$
\mathrm{d}=\mathrm{d}_{\mathscr{G}}: \mathrm{H}_{\mathscr{G}}^{1}(X) \longrightarrow \mathrm{L}_{2}(X), \quad f_{0} \mapsto f_{0}^{\prime}
$$


This operator is closed as operator from " 0 -forms" $\mathscr{H}_{0}:=\mathrm{L}_{2}(X)$ into " 1 -forms" $\mathscr{H}_{1}:=\mathrm{L}_{2}(X)$. The total Hilbert space for the boundary triple will be $\mathscr{H}:=\mathscr{H}_{0} \oplus \mathscr{H}_{1} \cong \mathrm{L}_{2}(X) \otimes \mathbb{C}^{2}$ and the elements are denoted by $f=f_{0} \oplus f_{1}$. Furthermore, we define

$$
\gamma_{0}: \mathrm{H}_{\mathscr{G}}^{1}(X) \longrightarrow \mathscr{G}, \quad f_{0} \mapsto \underline{f}_{0} .
$$

Using the notation of $[\mathrm{P} 07 \mathrm{~b}],\left(\mathscr{H}, \mathscr{G}, \gamma_{0}\right)$ is a first order boundary triple, i.e., d (the exterior derivative) is a closed operator from 0 -forms into 1 -forms, ker $\gamma_{0}$ is dense in $\mathscr{H}_{0}$ and the range ran $\gamma_{0}$ is dense in $\mathscr{G}$. Here, $\gamma_{0}$ is even surjective, i.e., the triple is not proper. We denote the restriction of $\mathrm{d}$ to $\operatorname{ker} \gamma_{0}=\mathrm{H}_{0}^{1}(X)$ by $\mathrm{d}_{0}$ and $\delta:=\mathrm{d}_{0}^{*}$, the divergence operator. Note that dom $\delta=\mathrm{H}_{\max }^{1}(X)$.

The maximal Dirac operator is now defined as

$$
D=D_{\mathscr{G}}^{\max }:=\left(\begin{array}{cc}
m & \delta \\
\mathrm{d}_{\mathscr{G}} & -m
\end{array}\right) \quad \text { with domain } \quad \operatorname{dom} D_{\mathscr{G}}^{\max }=\mathrm{H}_{\mathscr{G}}^{1}(X) \oplus \mathrm{H}_{\max }^{1}(X) .
$$

Here, we have restricted only the 0 -th component to the vertex space $\mathscr{G}$. The boundary operators in this case are defined as

$$
\begin{array}{ll}
\Gamma_{0}: \operatorname{dom} D \longrightarrow \mathscr{G}, & f \mapsto \gamma_{0} f_{0}=\underline{f}_{0} \\
\Gamma_{1}: \operatorname{dom} D \longrightarrow \mathscr{G}, & f \mapsto P \underline{f}_{1} .
\end{array}
$$

Lemma 6.1. Under the assumption (3.1) and with the above notation, $\left(\mathscr{G}, \Gamma_{0}, \Gamma_{1}\right)$ is a boundary triple for the maximal Dirac operator $D$ in $\mathscr{H}$.

Proof. By Lemma 4.3, $\mathrm{H}_{\mathscr{G}}^{1}(X)$ is complete, and one can easily see that the natural norm on $\mathrm{H}_{\mathscr{G}}^{1}(X) \oplus$ $\mathrm{H}_{\max }^{1}(X)$ and the graph norm on $\operatorname{dom} D$ are equivalent. In particular, $D$ is a closed operator. In order to show Green's formula (2.1a), use Corollary 4.5 to obtain

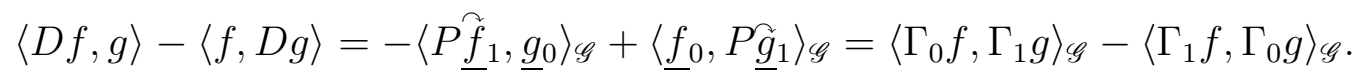

The surjectivity $(2.1 \mathrm{~b})$ is almost obvious, since we can prescribe the values $\underline{f}_{0}$ and $P \tilde{f}_{1}$ of the two components $f_{0}$ and $f_{1}$ independently. The density condition (2.1c) is easily seen from the density of the space of smooth functions with support away from the vertices.

The next lemma gives a relation between the Dirac and the Laplacian eigenspaces; its proof is a straightforward calculation:

Lemma 6.2. Let $w \neq \pm m, \mathscr{N}_{D}^{w}:=\operatorname{ker}(D-w)$ and $z:=w^{2}-m^{2}$, then

$$
\psi^{w}: \mathscr{N}_{\Delta}^{z} \longrightarrow \mathscr{N}_{D}^{w}, \quad f \mapsto\left(\begin{array}{c}
f \\
\frac{1}{w+m} \mathrm{~d} f
\end{array}\right)
$$

is a topological isomorphism.

Lemma 6.3. The Krein $\Gamma$-field of the above boundary triple is given by

$$
\beta_{D}(w)=\psi^{w} \beta_{\Delta}\left(w^{2}-m^{2}\right)
$$

where $\beta_{\Delta}$ is the Krein $\Gamma$-field associated to the boundary triple for the Laplacian given in Lemma 5.4.

Proof. It is a straightforward calculation to check that $f=\beta_{D}(w) F$ fulfills $D f=w f$ and $\Gamma_{0} f=$ $\underline{f}_{0}=F$ and similarly, $\beta_{D}(w) \Gamma_{0} f=f$ if $f \in \mathscr{N}_{D}^{w}$.

Combining Lemmas 6.2-6.3 we immediately obtain:

Lemma 6.4. The Krein Q-function associated to the given boundary triple for $D$ is

$$
Q_{D}(w):=\Gamma_{1} \beta_{D}(w)=\frac{1}{w+m} Q_{\Delta}\left(w^{2}-m^{2}\right),
$$

where $Q_{\Delta}$ denotes the Krein Q-function associated to the Laplace-boundary triple given in Lemma 5.5. 
Let $D_{0}$ be $D$ restricted to $\operatorname{ker} \Gamma_{0}$, i.e., $\operatorname{dom} D_{0}=\mathrm{H}_{0}^{1}(X) \oplus \mathrm{H}_{\max }^{1}(X)$. It is easily seen that $D_{0}$ is self-adjoint and that

$$
D_{0}^{2}=\bigoplus_{e}\left(\left(\Delta_{I_{e}}^{\mathrm{D}}+m^{2}\right) \oplus\left(\Delta_{I_{e}}^{\mathrm{N}}+m^{2}\right)\right)
$$

i.e., $\sigma\left(D_{0}^{2}\right)$ consists of the union of all Neumann spectra on the intervals $I_{e}$ shifted by $m^{2}$ (note that the Dirichlet spectrum of $I_{e}$ differs from the Neumann spectrum of $I_{e}$ only by 0 ). In particular,

$$
\sigma\left(D_{0}\right)=\left\{ \pm \sqrt{\left(\frac{\pi k}{\ell_{e}}\right)^{2}+m^{2}} \mid e \in E, k=0,1, \ldots\right\}
$$

and if all lengths $\ell_{e}$ are equal to 1 then

$$
\Sigma_{m}:=\sigma\left(D_{0}\right)=\left\{ \pm \sqrt{(\pi k)^{2}+m^{2}} \mid k=0,1, \ldots\right\} .
$$

We will not consider the exceptional values $\sigma\left(D_{0}\right)$ in the next theorem (see Remark 5.8 (iv)).

Let $M$ be a self-adjoint, bounded operator in $\mathscr{G}$. We denote by $D_{(\mathscr{G}, M)}$ the restriction of $D$ to the domain

$$
\operatorname{dom} D_{(\mathscr{G}, M)}:=\left\{f \in \mathrm{H}_{\mathscr{G}}^{1}(X) \oplus \mathrm{H}_{\max }^{1}(X) \mid \widetilde{P \underline{f}_{1}}=M \underline{f}_{0}\right\} .
$$

Note that $D_{(\mathscr{G}, M)}=D^{M}$ in the notation of Definition 2.4. As in Remark 5.6 one can check that the data $(\mathscr{G}, M)$ already cover all self-adjoint realisations of the Dirac operator; see also [BT90, BH03] for different parametrisations.

Again, we can apply Theorem 2.6 to our situation:

Theorem 6.5. Assume the lower bound on the edge lengths (3.1).

(i) For $w \notin \sigma\left(D_{0}\right)$ we have the relation between the eigenspaces

$$
\operatorname{ker}\left(D_{(\mathscr{G}, M)}-w\right)=\beta(w) \operatorname{ker}\left(Q_{\Delta}\left(w^{2}-m^{2}\right)-(w+m) M\right) .
$$

(ii) For $w \notin \sigma\left(D_{(\mathscr{G}, M)}\right) \cup \sigma\left(D_{0}\right)$ we have $0 \notin \sigma\left(Q_{D}(w)-M\right)$ and Krein's resolvent formula

$$
\left(D_{(\mathscr{G}, M)}-w\right)^{-1}=\left(\Delta_{0}-w\right)^{-1}-\beta_{D}(w)\left(Q_{D}(w)-M\right)^{-1}\left(\beta_{D}(\bar{w})\right)^{*}
$$

holds.

(iii) We have the spectral relation

$$
\sigma_{\bullet}\left(D_{(\mathscr{G}, M)}\right) \backslash \sigma\left(D_{0}\right)=\left\{\mu \in \mathbb{C} \backslash \sigma\left(D_{0}\right) \mid 0 \in \sigma_{\bullet}\left(Q_{\Delta}\left(\mu^{2}-m^{2}\right)-(\mu+m) M\right)\right\} .
$$

In particular, for an equilateral graph (i.e., $\ell_{e}=1$ ) and $\mu \notin \Sigma_{m}$, we have $\mu \in \sigma_{\bullet}\left(D_{(\mathscr{G}, M)}\right)$ iff

$$
0 \in \sigma_{\bullet}\left(\triangle_{\mathscr{G}}-\left(\sin _{1} \sqrt{\mu^{2}-m^{2}}\right)(\mu+m) M-\left(1-\cos \sqrt{\mu^{2}-m^{2}}\right)\right),
$$

where $\triangle_{\mathscr{G}}$ is the discrete Laplacian associated to the vertex space $\mathscr{G}$ (see Definition 3.7) and where $\bullet \in\{\emptyset, \mathrm{pp}$, disc, ess $\}$. Furthermore, the multiplicity of an eigenspace is preserved.

(iv) Assume that the graph is equilateral, and additionally, that $M=M_{0}$ id for some constant $M_{0} \in \mathbb{R}$, then for $\mu$ in a connected component of $\mathbb{R} \backslash \Sigma_{m}$, i.e., a spectral gap for $D_{0}$, we have $\mu \in \sigma_{\bullet}\left(D_{(\mathscr{G}, M)}\right)$ iff

$$
\left(\sin _{1} \sqrt{\mu^{2}-m^{2}}\right)(\mu+m) M_{0}+\left(1-\cos \sqrt{\mu^{2}-m^{2}}\right) \in \sigma_{\bullet}\left(\triangle_{\mathscr{G}}\right)
$$

for all spectral types, namely, $\bullet \in\{\emptyset$, pp, disc, ess, ac, sc, p $\}$. Again, the multiplicity of an eigenspace is preserved.

Let us illustrate the above result in a special case:

Example 6.6. If the operator $M=0$, then we see from Theorem 6.5 (iii) that $\sigma\left(D_{(\mathscr{G}, 0)}\right)$ is symmetric, i.e., $\mu \in \sigma\left(D_{(\mathscr{G}, 0)}\right)$ iff $-\mu \in \sigma\left(D_{(\mathscr{G}, 0)}\right)$. Moreover,

$$
D_{(\mathscr{G}, 0)}=D^{0}=\left(\begin{array}{cc}
m & \mathrm{~d}_{\mathscr{G}}^{*} \\
\mathrm{~d}_{\mathscr{G}} & -m
\end{array}\right)
$$


and the domain dom $D^{0}=\operatorname{dom}_{\mathscr{G}} \oplus \operatorname{dom~}_{\mathscr{G}}^{*}=\mathrm{H}_{\mathscr{G}}^{1}(X) \oplus \mathrm{H}_{\mathscr{G}_{\perp}}^{1}(X)$ (cf. Corollary 4.5) is decoupled. Furthermore,

$$
D_{(\mathscr{G}, 0)}^{2}=\left(D^{0}\right)^{2}=\left(\begin{array}{cc}
\Delta_{(\mathscr{G}, 0)}+m^{2} & 0 \\
0 & \Delta_{\left(\tilde{G}^{\perp}, 0\right)}+m^{2}
\end{array}\right),
$$

where $F \in \hat{\mathscr{G}}^{\perp}$ iff $\hat{F} \in \mathscr{G}^{\perp}=\mathscr{G}^{\max } \ominus \mathscr{G}$, i.e., $P \hat{F}=0$. In particular, the two components are decoupled. Moreover,

$$
\Delta_{(\mathscr{G}, 0)}=\mathrm{d}_{\mathscr{G}}^{*} \mathrm{~d} \mathscr{G} \quad \text { and } \quad \Delta_{\left(\mathscr{G}_{\perp} \perp 0\right)}=\mathrm{d}_{\mathscr{G}} \mathrm{d}_{\mathscr{G}}^{*}
$$

and one can also use supersymmetry in order to analyse the spectrum (see e.g. [P07a]).

Note that if we want the components of the functions in the self-adjoint operator domain dom $D_{(\mathscr{G}, 0)}$ to be invariant under permutation, then the invariance would enforce that $\mathscr{G}=\hat{\mathscr{G}}^{\perp}$, i.e., $\operatorname{dim} \mathscr{G}=\operatorname{deg} v-\operatorname{dim} \mathscr{G}$, i.e, $\operatorname{deg} v=0$.

\section{DiRAC OPERATORS With SYMMETRIC COMPONENTS}

Here, we would like to consider Dirac operators $D$ for which the domain of $D$ is invariant under permutation of the components. In general, if we want that $D$ is self-adjoint (i.e., of the form $\left.D=D_{(\mathscr{G}, M)}\right)$, then the components are invariant only for very special spaces $\mathscr{G}$ and operators $M$. In particular, $D_{(\mathscr{G}, 0)}$ (the "energy-independent" case, cf. Remark 4.8 (iii)) never has invariant components (see Example 6.6 above). Therefore we have to treat non-self-adjoint realisations of $D$.

Let $\mathscr{H}:=\mathrm{L}_{2}(X) \oplus \mathrm{L}_{2}(X) \cong \mathrm{L}_{2}(X) \otimes \mathbb{C}^{2}$ and let $\widetilde{D}=\widetilde{D}_{\mathscr{G}}^{\max }$ act formally as in Section 6 , but now with domain

$$
\operatorname{dom} \widetilde{D}:=\mathrm{H}_{\mathscr{G}}^{1}(X) \otimes \mathbb{C}^{2}
$$

for a fixed vertex space $\mathscr{G}$ with projection $P$ in $\mathscr{G}^{\max }$. Note that again the adjoint $\widetilde{D}^{*}$ acts formally as $D$, but on the domain $\mathrm{H}_{\widetilde{G}_{\perp}}^{1}(X) \otimes \mathbb{C}^{2}$.

Denote by $\widetilde{D}_{0}$ be the Dirac operator $\widetilde{D}$ for the minimal vertex space, i.e., the restriction of $\widetilde{D}$ onto dom $\widetilde{D}_{0}=\mathrm{H}_{0}^{1}(X) \otimes \mathbb{C}^{2}$. The adjoint $\widetilde{D}_{0}^{*}$ is defined on $\mathrm{H}_{\max }^{1}(X) \otimes \mathbb{C}^{2}$.

In order to analyse the non-self-adjoint operator $\widetilde{D}$, we consider its self-adjoint "Laplacian" $\widetilde{D}^{*} \widetilde{D}$. We first start with the following "maximal Laplacian", namely with

$$
\widetilde{\Delta}:=\widetilde{D}_{0}^{*} \widetilde{D} \quad \text { with domain } \quad \operatorname{dom} \widetilde{\Delta}=\mathrm{H}_{\mathscr{G}}^{2}(X) \otimes \mathbb{C}^{2} .
$$

Note that $\widetilde{\Delta}$ formally acts in each component as $\Delta+m^{2}$. The boundary space for the boundary triple will now be

$$
\widetilde{\mathscr{G}}:=\mathscr{G} \oplus \mathscr{G} \cong \mathscr{G} \otimes \mathbb{C}^{2} .
$$

The boundary operators in this case are defined as

$$
\begin{array}{ll}
\widetilde{\Gamma}_{0}: \operatorname{dom} \widetilde{\Delta} \longrightarrow \widetilde{\mathscr{G}}, & f \mapsto \underline{f}:=\left(\underline{f}_{0} \oplus \underline{f}_{1}\right) \\
\widetilde{\Gamma}_{1}: \operatorname{dom} \widetilde{\Delta} \longrightarrow \widetilde{\mathscr{G}}, & f \mapsto P \tilde{f}^{\prime}:=\left(P \underline{f}_{0}^{\prime} \oplus P \underline{f}_{1}^{\prime}\right)
\end{array}
$$

As before, it is a simple exercise to check that $\left(\widetilde{\mathscr{G}}, \widetilde{\Gamma}_{0}, \widetilde{\Gamma}_{1}\right)$ is a boundary triple for $\widetilde{\Delta}$ (similar to the arguments of Lemma 5.3).

Krein's $\Gamma$-field here is given by

$$
\widetilde{\beta}(z): \widetilde{\mathscr{G}} \longrightarrow \widetilde{\mathscr{N}^{z}}
$$

where $\widetilde{f}:=\widetilde{\beta}(z) \widetilde{F}$ is formally given as in Lemma 5 .4, but with $F$ replaced by the $\mathbb{C}^{2}$-valued vertex space element $\widetilde{F} \in \widetilde{\mathscr{G}}$ and with $z$ replaced by $z-m^{2}$. As before, Krein's Q-function is defined as $\widetilde{Q}(z)=\widetilde{\Gamma}_{1} \widetilde{\beta}(z)$. On an equilateral graph, we have

$$
\widetilde{Q}(z)=\frac{1}{\sin _{1} \sqrt{z-m^{2}}}\left(\begin{array}{cc}
\Delta_{\mathscr{G}}-\left(1-\cos \sqrt{z-m^{2}}\right) & 0 \\
0 & \Delta_{\mathscr{G}}-\left(1-\cos \sqrt{z-m^{2}}\right)
\end{array}\right)
$$


for $z \notin \Sigma^{\mathrm{D}}+m^{2}=\left\{(\pi k)^{2}+m^{2} \mid k=1,2, \ldots\right\}$.

Let $\widetilde{M}$ be a self-adjoint, bounded operator on $\widetilde{\mathscr{G}}$. Denote by $\widetilde{\Delta} \widetilde{M}$ the self-adjoint restriction of $\widetilde{\Delta}$ defined on

$$
\operatorname{dom} \widetilde{\Delta}^{\widetilde{M}}:=\left\{\tilde{f} \in \mathrm{H}_{\mathscr{G}}^{1}(X) \otimes \mathbb{C}^{2} \mid \widetilde{\Gamma}_{1} \widetilde{f}=\widetilde{M} \widetilde{\Gamma}_{0} \widetilde{f}\right\} .
$$

For shortness, we cite only the spectral relation of Krein's theorem in the equilateral case. The other assertions of Theorem 2.6 can easily be extracted also for this case.

Theorem 7.1. For an equilateral metric graph we have

$$
\lambda \in \sigma\left(\widetilde{\Delta}^{\widetilde{M}}\right) \Leftrightarrow 0 \in \sigma\left(\left(\triangle_{\mathscr{G}}-\left(1-\cos \sqrt{\lambda-m^{2}}\right)\right) \otimes \operatorname{id}_{\mathbb{C}^{2}}-\left(\sin _{1} \sqrt{\lambda-m^{2}}\right) \widetilde{M}\right) .
$$

for $\lambda \notin \Sigma^{\mathrm{D}}+m^{2}$.

Our aim now is to find self-adjoint restrictions corresponding to $\widetilde{D}^{*} \widetilde{D}$ : Remember that dom $\widetilde{D}^{*}=$ $\mathrm{H}_{\tilde{c} \perp}^{1}(X) \otimes \mathbb{C}^{2}$, i.e, $\widetilde{f} \in \operatorname{dom} \widetilde{D}^{*} \widetilde{D}$ iff $\widetilde{f} \in \operatorname{dom} \widetilde{D}$ and $\widetilde{D} \widetilde{f} \in \operatorname{dom} \widetilde{D}^{*}$, but this means that $\widetilde{f} \in$ $\mathrm{H}_{\max }^{2}(X) \otimes \mathbb{C}^{2}$ and

Moreover, $\underline{\tilde{D} \tilde{f}} \in \hat{\mathscr{G}}^{\perp} \otimes \mathbb{C}^{2}$ iff

$$
\underline{\tilde{f}} \in \tilde{\mathscr{G}} \quad \text { and } \quad \underline{\widetilde{D} \tilde{f}} \in \hat{\mathscr{G}}^{\perp} \otimes \mathbb{C}^{2}
$$

$$
P(\underline{\tilde{\mathbb{N}}} \underline{\widetilde{D} \tilde{f}})=\widetilde{\mathbb{1}}\left(\begin{array}{cc}
m & 0 \\
0 & -m
\end{array}\right) \widetilde{\Gamma}_{0} \widetilde{f}+\left(\begin{array}{cc}
0 & -1 \\
1 & 0
\end{array}\right) \widetilde{\Gamma}_{1} \widetilde{f}=0
$$

where $\tilde{\mathbb{1}}$ denotes multiplication with \pm 1 depending whether $v=\partial_{ \pm} e$. Therefore, we have

$$
\widetilde{D}^{*} \widetilde{D}=\widetilde{\Delta}^{\widetilde{M}} \quad \text { for } \quad \widetilde{M}=m \widetilde{\mathbb{1}}\left(\begin{array}{ll}
0 & 1 \\
1 & 0
\end{array}\right) .
$$

In order to calculate the spectrum of $\widetilde{D}^{*} \widetilde{D}$, we need the following lemma:

Lemma 7.2. Assume that

$$
\widetilde{B}=\left(\begin{array}{cc}
A & b \\
b & A
\end{array}\right)
$$

in $\widetilde{\mathscr{G}}=\mathscr{G} \oplus \mathscr{G}$ where $A$ is a self-adjoint, bounded operator in $\mathscr{G}$ and $b \in \mathbb{R}$. For simplicity only, we assume that $A$ has pure point spectrum. Then

$$
0 \in \sigma(\widetilde{B}) \quad \Leftrightarrow \quad \exists \eta_{1}, \eta_{2}: \eta_{1} \eta_{2}=b^{2} .
$$

Proof. Let $\mathscr{G}=\bigoplus_{k} \mathbb{C} \varphi_{k}$ be a decomposition into eigenspaces of $A$. If $\widetilde{f}=\sum_{j, k} f_{0, j} \varphi_{j} \oplus f_{1, j} \varphi_{k}$ for coefficients $f_{p, j} \in \mathbb{C}$, then $\widetilde{f} \in \operatorname{ker} \widetilde{B}$ is equivalent to

$$
\left(\begin{array}{cc}
\eta_{j} & b \\
b & \eta_{k}
\end{array}\right)\left(\begin{array}{l}
f_{0, j} \\
f_{1, k}
\end{array}\right)=0
$$

for all $j, k$; i.e., we have a non-trivial solution iff there exist $j, k$ such that the determinant of the matrix vanishes, i.e., iff $\eta_{j} \eta_{k}=b^{2}$. The converse statement can be shown similarly.

Combining the previous lemma with Theorem 7.1 yields:

Theorem 7.3. For a finite, equilateral metric graph we have the following spectral relation for the Dirac operator with prescribed vertex space $\mathscr{G} \otimes \mathbb{C}^{2}$ for the vertex values, namely, for $\lambda \notin \Sigma^{\mathrm{D}}+m^{2}$, we have $\lambda \in \sigma\left(\widetilde{D}^{*} \widetilde{D}\right)$ iff there exist $\eta_{1}, \eta_{2} \in \sigma\left(\triangle_{\mathscr{G}}\right)$ such that

$$
\left(\eta_{1}-1+\cos \sqrt{\lambda-m^{2}}\right)\left(\eta_{2}-1+\cos \sqrt{\lambda-m^{2}}\right)=m^{2}\left(\sin _{1} \sqrt{\lambda-m^{2}}\right)^{2} .
$$

Note that the orientation in the matrix coefficient $b=m \tilde{1} \sin _{1} \sqrt{\lambda-m^{2}}$ disappears since only $b^{2}$ counts in Lemma 7.2 . 
Example 7.4. In order to keep this article at a reasonable size, we only sketch a simple consequence: Let $m=1$ and $\lambda \notin \Sigma^{\mathrm{D}}+1$. Then a value $\lambda$ is in the spectrum of $\sigma\left(\widetilde{D}^{*} \widetilde{D}\right)$ iff one can find values $\alpha_{p} \in \sigma\left(\Delta_{\mathscr{G}}\right)+1=: I$ such that

$$
\left(\alpha_{1}+\cos \mu\right)\left(\alpha_{2}+\cos \mu\right)=\frac{\sin \mu}{\mu}
$$

has a solution for $\mu=\sqrt{\lambda-1}$. In particular, $\lambda \geq 1$, and $\lambda$ is not in the spectrum iff the curve in $\alpha_{1}$ and $\alpha_{2}$ (for $\mu$ fixed) has empty intersection with the set $I \times I$.

\section{Conclusion}

For equilateral graphs, we showed a spectral relation and a resolvent formula for Laplacian and Dirac operators on a metric graph with an appropriately defined discrete Laplacian on the space of vertex values $\mathscr{G}$. Here, we indicate further directions to be analysed, which may also be interesting of its own:

- Since - at least in the equilateral case and for finite graphs - all self-adjoint Laplace and Dirac metric graph operators are completely understood by the generalised discrete Laplacians $\triangle_{\mathscr{G}}$, one should systematically analyse $\triangle_{\mathscr{G}}$ for general vertex spaces, e.g. the spectrum, the resolvent and a decomposition of $\mathscr{G}$ and $\Delta_{\mathscr{G}}$ into "irreducible" blocks (see [P07c, Def. 2.4]).

- In order not to obscure the basic ideas by too many details, we considered only the Laplacian, i.e., the free Hamiltonian on each interval $I_{e}$. Our results can easily be generalised to the case, when the operator acts as $-(\cdot)_{e}^{\prime \prime}+q_{e}$ on each edge; basically, one has to replace the explicit fundamental solutions (5.2) by the appropriate fundamental solutions of the ODE $-f_{e}^{\prime \prime}+q_{e} f_{e}=z f_{e}$. The edge operator enters into the spectral relation via Hill's discriminant only, i.e., the behaviour of the edge operator is completely decoupled from the combinatorial structure. Actually, Pankrashkin [Pa07, Pa06] (see also [BGP06]) uses an even more general setting, replacing the simple Laplacian on an edge by any type of abstract edge operator (the same for each edge) with defect index $(2,2)$.

- Our analysis of metric graph operators can be used in order to analyse periodic problems via Floquet theory. In particular, one can check whether the metric graph operators have a spectral gap or not (see Remark $5.8(\mathrm{v})$ ). A systematic analysis of periodic generalised discrete Laplacians would be of interest.

- One should analyse in more detail the relation between the different spectral types also for the operator of Section 7 or more general types of vector-valued differential operators on a metric graph.

\section{REFERENCES}

[vB85] J. von Below, A characteristic equation associated to an eigenvalue problem on $C^{2}$-networks, Linear Algebra Appl. 71 (1985), 309-325.

[BCFK06] G. Berkolaiko, R. Carlson, St. A. Fulling, and P. Kuchment (eds.), Quantum graphs and their applications, Contemp. Math., vol. 415, Providence, RI, AMS, 2006.

[BH03] J. Bolte and J. Harrison, Spectral statistics for the Dirac operator on graphs, J. Phys. A 36 (2003), no. 11, 2747-2769.

[BGP06] J. Brüning, V. Geyler, and K. Pankrashkin, Spectra of self-adjoint extensions and applications to solvable Schrödinger operators, to appear in Rev. Math. Phys. (arXiv:math-ph/0611088) (2006).

[BGP07]_, Cantor and band spectra for periodic quantum graphs with magnetic fields, Comm. Math. Phys. 269 (2007), no. 1, 87-105.

[BT90] W. Bulla and T. Trenkler, The free Dirac operator on compact and noncompact graphs, J. Math. Phys. 31 (1990), no. 5, 1157-1163.

[CW05] D. I. Cartwright and W. Woess, The spectrum of the averaging operator on a network (metric graph), Preprint arXiv : math.FA/0509595 (2005). 
[Ca97] C. Cattaneo, The spectrum of the continuous Laplacian on a graph, Monatsh. Math. 124 (1997), no. 3, $215-235$.

[DeN00] B. Dekoninck and S. Nicaise, The eigenvalue problem for networks of beams, Linear Algebra Appl. 314 (2000), no. 1-3, 165-189.

[DHMdS06] V. Derkach, S. Hassi, M. Malamud, and H. de Snoo, Boundary relations and their Weyl families, Trans. Amer. Math. Soc. 358 (2006), no. 12, 5351-5400.

[EP07] P. Exner and O. Post, Quantum networks modelled by graphs, Preprint arXiv:0706.0481 (2007).

[E97] P. Exner, A duality between Schrödinger operators on graphs and certain Jacobi matrices, Ann. Inst. H. Poincaré Phys. Théor. 66 (1997), no. 4, 359-371.

[FKW07] S. Fulling, P. Kuchment, and J. H. Wilson, Index theorems for quantum graphs, J. Phys. A 40 (2007), no. $47,14165-14180$.

[Ha00] M. Harmer, Hermitian symplectic geometry and extension theory, J. Phys. A 33 (2000), no. 50, 91939203.

[HP06] P. Hislop and O. Post, Exponential localization for radial random quantum trees, Preprint math-ph/0611022 (2006).

[KoPS07] V. Kostrykin, J. Potthoff, and R. Schrader, Heat kernels on metric graphs and a trace formula, Contemp. Math., vol. 447, AMS, Providence, RI, 2007, pp. 175-198.

[KoS99] V. Kostrykin and R. Schrader, Kirchhoff's rule for quantum wires, J. Phys. A 32 (1999), no. 4, 595-630.

[KoS06] _ Laplacians on metric graphs: eigenvalues, resolvents and semigroups, Contemp. Math., vol. 415, AMS., Providence, RI, 2006, pp. 201-225.

[K04] P. Kuchment, Quantum graphs: I. Some basic structures, Waves Random Media 14 (2004), S107-S128.

[K05] , Quantum graphs. II. Some spectral properties of quantum and combinatorial graphs, J. Phys. A 38 (2005), no. 22, 4887-4900.

[KP07] P. Kuchment and O. Post, On the spectra of carbon nano-structures, Comm. Math. Phys. 275 (2007), no. $3,805-826$.

[Pa06] K. Pankrashkin, Spectra of Schrödinger operators on equilateral quantum graphs, Lett. Math. Phys. $7 \mathbf{7}$ (2006), no. 2, 139-154.

[Pa07] $\quad$ Reduction of quantum graphs to tight-binding Hamiltonians, talk at the Isaac Newton Institute (INI), Cambridge (03-04-2007).

[P06] O. Post, Spectral convergence of quasi-one-dimensional spaces, Ann. Henri Poincaré 7 (2006), no. 5, 933-973.

[P07a] , First order approach and index theorems for discrete and metric graphs, Preprint arXiv: $0708.3707(2007)$.

[P07b] _ First order operators and boundary triples, Russ. J. Math. Phys. 14 (2007), no. 2, $482-492$.

[P07c] Spectral analysis of metric graphs and related spaces, Preprint arXiv:0712.1507 (2007).

[V63] M. I. Vishik, On general boundary problems for elliptic differential equations., Am. Math. Soc., Transl., II. Ser. 24 (1963), 107-172 (English. Russian original from 1952).

Institut für Mathematik, Humboldt-Universität Zu Berlin, Rudower Chaussee 25, 12489 Berlin, GERMANY

E-mail address: post@math.hu-berlin.de 